\title{
Evaluation of staring episodes in children
}

\author{
Arif Khan, ${ }^{1}$ Nahin Hussain, ${ }^{1}$ William P Whitehouse ${ }^{2}$
}

- Additional supplementary files are published online only. To view these files please visit the journal online (http://dx.doi.org/ 10.1136/archdischild-2011301111).

${ }^{1}$ Department of Paediatric Neurology, Leicester Royal Infirmary, University Hospitals of Leicester NHS Trust, Leicester, Leicestershire, UK

${ }^{2}$ Department of Paediatric Neurology, University of Nottingham, Nottingham Children's Hospital, Nottingham University Hospital's NHS Trust, Queens Medical Centre, Nottingham, UK

\section{Correspondence to} Dr Arif Khan, Department of Paediatric Neurology, Leicester Royal Infirmary, Infirmary Square, Leicester LE1 5WW, UK, khangoa@hotmail.com

Accepted 14 July 2012 Published Online First 31 August 2012

\section{Introduction}

Staring episodes are a frequent reason for referral to paediatrics, as parents and teachers fear the possibility of seizures. Staring episodes in childhood are common and a challenging clinical problem. The difficulty is in differentiating between epileptic and non-epileptic phenomenon at the first assessment. Clear description of the event, preferably by a first-hand eye witness, along with clinicians' clinical experience will usually lead to a correct categorisation of the episodes. Rarely, a clinician will be able to actually observe the phenomenon; therefore a video recording of an event is invaluable.

The differential diagnosis of staring spells in children can be broadly categorised into absence seizures ('typical' in a 'normal' child and 'atypical' in a child with developmental problems), complex partial seizures (CPS), daydreaming and childhood preoccupation. To achieve an accurate diagnosis a comprehensive approach including a systematic clinical assessment and judicious use of investigations is necessary.

However, it should be remembered that as paediatricians we work with children of varying ages and maturity levels, in addition to the underlying neurological impairments that may exist. There is often a lack of information, description and insight into these events. Therefore, the proportion of 'misdiagnosis' in epilepsy in this population (infants, young children and neurologically impaired children) is substantial. $^{12}$

A simple approach and categorisation of these events is offered here to help the clinician in making an accurate diagnosis.

Staring episodes in children can be broadly divided into non-epileptic and epileptic events.

\section{Non-epileptic staring episodes Daydreaming}

Daydreaming in children is common. Those children who are referred for evaluation are more likely to have comorbid conditions such as attention deficit hyperactivity disorder (ADHD), learning difficulties and Tourette syndrome. These episodes usually occur during periods of inactivity or when a child finds the activity less interesting. Children during daydreaming tend to ignore verbal stimulation, but usually respond to tactile stimulation. ${ }^{3}$ However, in children with learning difficulties and/ or developmental delay it may be difficult to interrupt these events. The clue to the diagnosis of all these events is that they can be interrupted by touch or tickle and that they do not interrupt otherwise interesting activities.

\section{Childhood preoccupation}

Preoccupation occurs when the child is engrossed in a task. The child appears vacant or glazed (occasionally the eyes may be closed). They could have a glazed expression doing their homework and humming. The length of the episode can be variable depending on the surrounding distraction and they can be stopped by verbal stimulation. ${ }^{4}$ Gratification disorder previously referred to as infantile masturbation and benign idiopathic infantile dyskinesia is a well-recognised phenomenon in infants. ${ }^{5}$ This behaviour is not akin to the typical manual genital stimulation behaviour as in older age. Parents report their child staring, grunting and rocking to and fro with crossed legs. This condition is often under-recognised or misdiagnosed as epilepsy. Additional features include (1) onset after the age of 3 months and before 3 years (2) facial flushing with diaphoresis (3) no alteration of consciousness (4) cessation with distraction (5) normal examination and laboratory studies (6) pressure on the perineum with characteristic posturing of lower extremities. ${ }^{6}$ Observation of the episode in person or video is sufficient to make a diagnosis. No further investigations or treatment is 
needed. Parental acknowledgement is at times difficult or delayed. However, clear explanation of the phenomenon with evidence of distractibility should help.

\section{Migraine-like events}

Another entity, which has been described in some children, is the 'Alice in Wonderland' phenomenon. These events are thought to be migraine-related. This peculiar syndrome of visual and interpretive distortions of body and environmental surroundings is well recognised in children. ${ }^{78}$ This may last for $15-20 \mathrm{~min}$ and is then followed by throbbing headaches. During this event, the child may appear to stare, as they are either scared or perplexed by this experience.

Children can suffer from headaches (either migraine and/or other type of headache) and epilepsy, either as two different pathologies or having a common underlying pathology. Staring episodes may be a feature of these episodes. In these cases, the headache occurs at a different time to the seizure (interictal headache). However, headaches sometimes occur simultaneously with or very close in time to the seizure. Headaches that occur at the time of epileptic seizure are known as 'ictal epileptic headaches' or as 'hemicrania epileptica'. One that precedes a seizure is known as 'preictal headache' and one that follows a seizure is known as 'postictal headache'. There is a particular type of preictal headache, known as 'migralepsy', which occurs during or just after a migraine aura. This entity although described is extremely rare. ${ }^{9}$ Children during these headache episodes tend to stare and remain unresponsive.

\section{Other less common causes}

Cyclical vomiting, parasomnias and psychogenic seizures could also have staring episodes as one of their features. However, they are usually associated with other more predominant symptoms like vomiting, nocturnal sleep-related events and inconsistency respectively.

\section{Epileptic staring episodes}

For children referred for staring episodes, only absence and complex partial seizures need to be considered in the epileptic category. The differentiation between these two seizure types is detailed in table 1 .

Table 1 Differentiation between absence and complex partial seizures

\begin{tabular}{lll}
\hline Features & $\begin{array}{l}\text { Absence } \\
\text { seizures }\end{array}$ & $\begin{array}{l}\text { Complex partial } \\
\text { seizures }\end{array}$ \\
\hline Duration & $<20 \mathrm{~s}$ & $\geq 1 \mathrm{~min}$ \\
Aura & Absent & Present \\
Postictal drowsiness & Absent & Present \\
\hline
\end{tabular}

Adapted from Current Management in Child Neurology, Third Ed 2005.

\section{Absence seizures}

Absence seizures in childhood are often divided into two major categories: typical and atypical. The international league against epilepsy (ILAE) commission classification and terminology have simplified the classification of absence seizures as follows:

1. Absence seizures: Typical or atypical, each with distinct features (table 2)

2. Absences with special features: myoclonic absences and eyelid myoclonia.

Typical absences (previously known as petit mal) are brief (lasting seconds) generalised epileptic seizures of abrupt onset and abrupt termination. They have two essential components: (1) A clinical component manifesting with impairment of consciousness (absence) (2) An EEG component manifesting with generalised spike-slow-wave discharges of 3 or $4 \mathrm{~Hz}(>2.5 \mathrm{~Hz}) .^{10} 11$ Atypical absences are generalised epileptic seizures of inconspicuous start and termination with (1). Clinical symptoms of mild-to severe impairment of consciousness (absence), often significant change in tone with hypotonia or atonia, mild tonic or autonomic alterations (2). EEG discharges of slow spike-wave $(1-2.5 \mathrm{~Hz})$, which are often irregular and heterogeneous and may be mixed with fast rhythms. ${ }^{10-12}$

Myoclonic absences are rare. The mean age of onset is 7 years. The seizures have a sudden onset and ending, and usually occur soon after awakening.

Table 2 Differences between atypical and typical absence seizures

\begin{tabular}{|c|c|c|}
\hline Features & Atypical absences & Typical absences \\
\hline $\begin{array}{l}\text { Onset and } \\
\text { termination }\end{array}$ & Usually gradual & Abrupt \\
\hline Responsiveness & $\begin{array}{l}\text { Decreased but not } \\
\text { abolished }\end{array}$ & $\begin{array}{l}\text { Varies from mild to } \\
\text { severe }\end{array}$ \\
\hline Changes in tone & Usually pronounced & Usually mild \\
\hline Duration & $\begin{array}{l}\text { Usually long sometimes } \\
\text { minutes }\end{array}$ & $\begin{array}{l}\text { Usually brief; never } \\
>30-40 \mathrm{~s}\end{array}$ \\
\hline Postictal recovery & $\begin{array}{l}\text { Cognitive impairment } \\
\text { may persist }\end{array}$ & Immediately \\
\hline Interictal EEG & $\begin{array}{l}\text { Background often } \\
\text { abnormal with frequent } \\
\text { discharges of various } \\
\text { types }\end{array}$ & $\begin{array}{l}\text { Background usually } \\
\text { normal sometimes with } \\
\text { typical IGE discharges }\end{array}$ \\
\hline Ictal EEG & $\begin{array}{l}\text { Slow }(<2.5 \mathrm{~Hz}) \text { spike } \\
\text { and wave }\end{array}$ & $\begin{array}{l}\text { Fast }(>2.5 \mathrm{~Hz}) \text { spike } \\
\text { and slow wave }\end{array}$ \\
\hline $\begin{array}{l}\text { Normal } \\
\text { neurological and } \\
\text { mental state }\end{array}$ & Exceptional & As a rule \\
\hline $\begin{array}{l}\text { Other types of } \\
\text { seizures }\end{array}$ & $\begin{array}{l}\text { Atonic and tonic seizures } \\
\text { of symptomatic } \\
\text { generalised epilepsies }\end{array}$ & $\begin{array}{l}\text { Depend on IGE } \\
\text { syndrome (myoclonic } \\
\text { jerks, GTCS or both) }\end{array}$ \\
\hline Prognosis & Commonly bad & Commonly good \\
\hline
\end{tabular}

Adapted from Panayiotopoulos CP. A clinical Guide to Epileptic Syndromes and their treatment 2007.

IGE, idiopathic generalised epilepsy, GTCS, generalised tonic clonic seizures. 
Seizure duration ranges from less than $10 \mathrm{~s}$ to more than $2 \mathrm{~min}$. The myoclonus is rhythmic and usually involves the shoulders, arms and head. They may have perioral myoclonus, although eyelid myoclonus is rare. The EEG shows a typical bilateral $3 \mathrm{~Hz}$ spikewave or polyspike activity. ${ }^{3}$

In eyelid myoclonia with absences, absence follows the eyelid myoclonia while the eyelid jerking continues. Consciousness is mildly impaired and automatisms are not observed. Another symptom is the rare occurrence of the 'pleasurable' feeling during the absence, which may be related to 'self-induction' in some patients. ${ }^{13}$

Frontal onset absences constitute a specific subtype within the childhood absence epilepsies. Children with frontal onset of the EEG abnormalities frequently have complex absences and are more likely to have learning and behavioural problems. They are usually resistant to treatment. ${ }^{14}$

In 1985, the ILAE task force listed multiple epileptic syndromes defined by seizure type(s), age of onset, or seizure frequency, or a combination of two or more of these features. This 'syndromic' approach for epilepsy classification has since been maintained with few modifications. ${ }^{15}$ The purpose of classification of epilepsies into epileptic syndromes is to organise items according to their fundamental relationship. This will help clinicians make or attempt accurate diagnosis, which is important for proper treatment and prognosis of epilepsies.

ILAE has recognised absence seizures as manifestation of certain epileptic syndromes in children. These include Childhood Absence Epilepsy (CAE), Juvenile Absence Epilepsy (JAE) and Juvenile Myoclonic Epilepsy (JME).

- CAE: CAE is among the easiest, most straightforward and satisfying diagnosis in neurology. However, many children have a delayed diagnosis due to the non-alarming nature of the presentation. The absences in this syndrome may be too short to be recognised; they may not have prominent automatisms or autonomic changes to aid their detection. The EEG is essential to confirm the diagnosis as some partial epilepsies and daydreaming may have similar presentation. The clinical features of CAE are (1) onset between 4 and 10 years (2) normal neurological and developmental status (3) frequent brief absence seizures with abrupt and significant impairment of consciousness (4) EEG changes of generalised spike-and-wave complexes at $3 \mathrm{~Hz}$ lasting $4-20 \mathrm{~s}^{3}$. Hyperventilation test in children with CAE provokes absences in as many as $90 \%$ of those who suffer from it. The prognosis of CAE is excellent. Remission occurs before 12 years of age. Although $10 \%$ of patients may develop infrequent or solitary GTCS in adolescence or adult life. ${ }^{16} 17$
- JAE: The age ranges of JAE and CAE overlap, however their prognosis and treatment are different. JAE usually presents after 9 years of age but range is between 5 to 20 years. ${ }^{18} \mathrm{JAE}$ frequently evolves over time and includes other generalised seizures and myoclonus. Many JAE patients evolve into JME and therefore the treatment regimens for this syndrome should take into account these risks. Frequent and severe typical absences are the characteristics and defining seizures of JAE. In JAE automatisms and mild myoclonic elements of eyelid are common during absences. GTC seizures occur in $76 \%$ of patients mainly after awakening but these are usually infrequent. ${ }^{19}$ Myoclonic jerks occur in $15 \%-25 \%$ of patients but are infrequent and mild. They usually occur at mid-day rather than early morning (as in JME). ${ }^{20}$ The ictal EEG shows $3-4 \mathrm{~Hz}$ generalised polyspike wave discharges. JAE unlike CAE is a lifelong disorder but seizures can be controlled in the majority of patients.

- JME: JME can easily be misdiagnosed as JAE if the history of awakening myoclonus is not forthcoming. JME is confirmed once a history of early morning myoclonus, or presence of overt myoclonic or GTC seizures is obtained along with absences in the appropriate age group. In JME, absences begin in late childhood followed by myoclonus in early adolescence and GTC seizures in late adolescence. EEG shows characteristic 3-4 Hz generalised polyspike-and-wave discharges on a normal inter-ictal background. Most patients will need lifelong treatment with antiepileptic drugs.

\section{Complex partial seizures}

Although absences and complex partial seizures both involve staring and unresponsiveness, there are several important features which help differentiate these two seizure types (table 1). It is essential to differentiate between these two epileptic seizures as the investigations, treatment and prognosis are significantly different. Complex partial seizures may begin at any age from early infancy through adulthood. They tend to be longer than absence seizures, generally lasting more than $30 \mathrm{~s}$ (usually $1-3 \mathrm{~min}$ ). The postictal state is another difference between the two seizure types. After an absence seizure, the child will resume his prior activity. With complex partial seizures the child usually goes into an altered mental status (confusion, difficulty speaking, sleepiness or unusual emotions) lasting few minutes to few hours. EEG in complex partial seizures tends to show abnormal electrical activity localised to one area (focal epileptiform discharges). Following confirmation of complex partial seizures, neuroimaging is essential to rule out any localising lesion (MRI preferable). 


\section{Approach to staring spells in children}

In order to diagnose a staring episodic disorder accurately, the clinician needs to answer four questions and adopt a systematic approach (See figure 1).

1. Is it an epileptic or non-epileptic episode?

A detailed history, which includes clear first-hand eyewitness account of the episode is crucial to the diagnosis. The eyewitness account should include what happens before, during and after the episode, along with response to tactile stimulation (tactile interruptibility). Non-epileptic episodes can be interrupted with tactile stimulation, whereas epileptic episodes are not interrupted. Absences are situation independent, unpredictable and can disrupt activities, whereas daydreaming and childhood preoccupation are situation dependent, predictable and 'almost never occur when the child is physically active'. A parental questionnaire-based study of 40 children with staring spells (including children with absence epilepsy and non-epileptic staring) was undertaken to identify questions sensitive and specific for staring spells of epileptic or nonepileptic aetiology to increase the yield of history taking. They concluded that in children with normal interictal electroencephalography findings and without neurological disease, staring spells were most likely non-epileptic when parents reported preserved responsiveness to touch, body rocking, or initial identification by a teacher or health professional without limb twitches, upward eye movements, interruption of play, or urinary incontinence. In these children a diagnosis of non-epileptic staring spells can be made with reinforcement from long-term follow-up. ${ }^{21}$

2. If it is epileptic, what is the type of seizure?

A detailed description of the episode in the form of first-hand eyewitness account and video review should help to identify important features to help differentiate between absences and complex partial seizures. These include frequency, abruptness of onset and termination, duration of episode, lack of awareness, aura, automatism and postictal phase. Associated features like periocular and perioral myoclonia, myoclonic seizures, generalised tonic clonic seizures and atonic seizures should be explored.

Voluntary hyperventilation has been used as a test to provoke epileptic seizures since the phenomenon

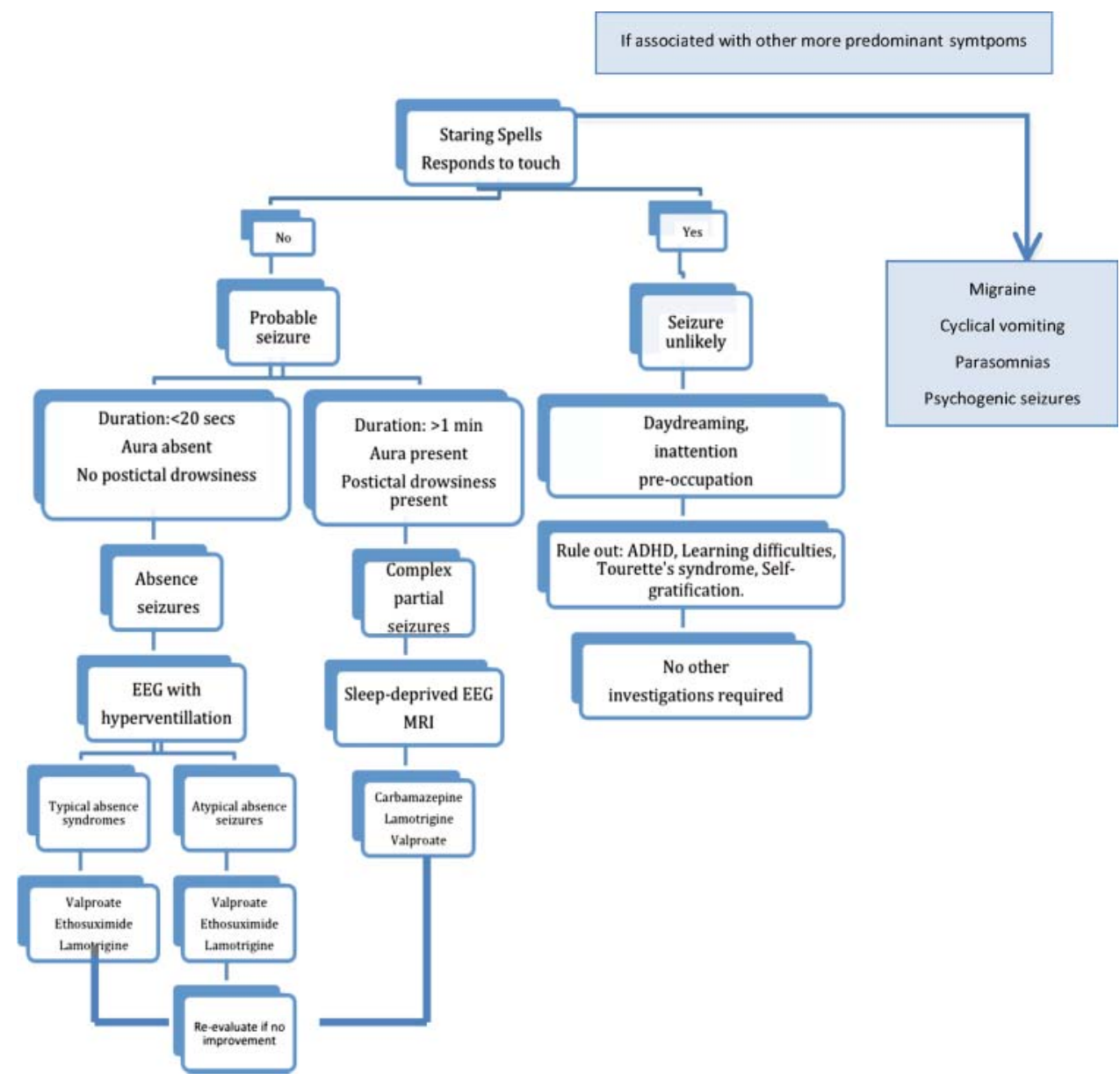

Figure 1 Approach to staring spells in children. ADHD, attention deficit hyperactivity disorder, EEG, MRI. Adapted from Dooley JM. Staring Spells. In: Current management in Child Neurology, Third edition. 
was first described in $1924 .{ }^{22}$ Typical absence seizures can easily be triggered by hyperventilation in majority of children with absence epilepsy. It is easily performed by asking the child to over-breathe for up to $3 \mathrm{~min}$ in an outpatient setting and can be diagnostic on clinical grounds. This could be achieved by asking them to blow a piece of paper or a windmill toy. Hyperventilation may elicit clinical seizure in as many as one half of patients with generalised epilepsy particularly in children with typical absences. ${ }^{23}$ However, in clinical practice if no event is triggered then we still go ahead with EEG and if an unresponsive stare with motor arrest with or without automatisms is triggered then an epilepsy is very likely but EEG is still needed to confirm absence seizures as opposed to focal seizures. All children with suspected seizures should have an EEG along with hyperventilation as a part of the protocol. When complex partial seizures are suspected a sleep-deprived EEG should be attempted as this may help provoke a seizure. EEG protocol for complex partial seizures should include EEG in awake, drowsy and stage II sleep states, as the chance of recording a focal epileptic discharge increases. Adding video recording to routine EEG for hospitalised children and outpatients increases the diagnostic yield. ${ }^{24}$ In addition, short-duration video-EEG lasting 2-8 h or more prolonged video-telemetry studies could be requested to evaluate less frequent events.

3. Can the seizures be categorised into a particular epileptic syndrome?

As discussed earlier, the clinician should have the knowledge of the various epileptic syndromes associated with absence seizures and also the manifestation of complex partial seizures. After having established the type of seizures (absence or complex partial seizures), the next step should be to establish a diagnosis of epileptic syndrome. The age of onset, presence of associated features, evolution of the seizure frequency over time and EEG features need to be taken into consideration.

4. What is the underlying cause?

MRI scan is usually not necessary if the history is suggestive of typical absence seizures but if the semiology is suggestive of complex partial seizures, then a MRI scan is indicated especially in the presence of focal neurological signs and/or developmental delay. The National Institute of Clinical Excellence (NICE) recommends MRI brain scan in all children diagnosed with epilepsy under 2 years of age and those who have any suggestion of a focal onset on history, examination or EEG (unless clear evidence of benign focal epilepsies). MRI should not be routinely requested when a diagnosis of idiopathic generalised epilepsy has been made. ${ }^{25}$

If complex partial seizures are present, neuroimaging in the form of MRI should be undertaken to explore any localising lesion in the brain. Associated comorbidities like ADHD, learning difficulties, Tourette's syndrome should be explored, as non-epileptic episodes are commonly associated with these conditions. Once a diagnosis of epileptic syndrome is established, no further investigations are required unless complex partial seizures are suspected or atypical absences are present.

\section{Management}

Children with daydreaming episodes do not require any intervention. Those with childhood preoccupation, once confirmed, would require parental reassurance. Sometimes, classroom modifications and home environment adaptation may be beneficial in reducing the frequency of these episodes. If comorbid conditions like ADHD, learning disability or Tourette's syndrome are identified, additional targeted interventions may be required.

The spectrum of motor activity in infantile selfgratification disorder is wide and therefore difficult to recognise. Many of these children undergo unwarranted investigations such as blood tests, metabolic screening, abdominal ultrasound and neuroimaging. Some of these are undertaken under general anaesthesia. Many of these children have received treatment with antiepileptic medications. ${ }^{26}{ }^{27}$ Careful interrogation and a video recording of the event are most helpful in understanding the nature of these events. The acknowledgement of the diagnosis by parents may not be immediate but clear explanation and reassurance is the key to their acceptance.

To ascertain the epileptic nature of events, parents should be advised to maintain a seizure diary to record a first-hand eyewitness account of what happens before, during and after the episodes. The diary should be reviewed on every clinic visit.

When epilepsy is confirmed, appropriate antiepileptic medication should be initiated.

It is essential to differentiate between absence seizures and complex partial seizures, as some of the antiepileptic medication used to treat the latter (carbamazepine) may worsen absence seizures.

Sodium valproate and ethosuximide are equally effective as monotherapy in treatment of $\mathrm{CAE}^{27}$ Many clinicians prefer sodium valproate as a first choice as unlike ethosuximide, it controls myoclonic jerks and generalised tonic clonic seizures, which may be occasionally associated with the absence seizures. However, these seizure types may not be of concern in CAE syndrome. It has good efficacy in atypical absence seizures as well. Occasionally, some clinicians due to its better tolerability prefer ethosuximide. Monotherapy with either valproate or ethosuximide controlled absences in 58\% and 53\% of children respectively. Lamotrigine is also an effective alternative for absence seizures but is less efficacious compared to valproate or ethosuximide. ${ }^{27}$ Moreover, skin 
hypersensitivity is a major adverse effect and therefore its slow titration over 8 weeks makes it less acceptable. Patients who do not respond to first monotherapy with either sodium valproate or ethosuximide should be tried on the other drug. If monotherapy fails then a combination of valproate and ethosuximide or lamotrigine may be tried.

For children with CPS, carbamazepine is the firstline antiepileptic drug (AED) used by most clinicians, although oxcarbazepine could have similar effectiveness without the potential of haematotoxicity or hepatotoxicity.

\section{Summary}

A thorough interrogation of the events, which includes response to touch or tickling and association with 'boredom' should be undertaken. Analysis of video recording should be sought if direct observation is not possible. No further investigations are warranted to make a diagnosis of daydreaming or childhood preoccupation.

If the episodes are not terminated by stimulation and interrupt activities, they are more likely to be seizures. One should try and differentiate between absence seizures and complex partial seizures using eyewitness account, hyperventilation test and EEG. This differentiation is essential, to help make an appropriate choice of AEDs.

Contributors $\mathrm{NH}$ : concept and literature search; AK: write-up and manuscript production; WPW: final corrections.

\section{Competing interests None.}

Provenance and peer review Not commissioned; externally peer reviewed.

\section{References}

1. Donat JF, Wright FS. Episodic symptoms mistaken for seizures in the neurologically impaired child. Neurology 1990; 40:153-7.

2. Shuper A, Mimouni M. Problems of differentiation between epilepsy and non-epileptic paroxysmal events in the first year of life. Arch Dis Child 1995;73:342-4.

3. Dooley JM. Staring spells. In: Maria BL, ed. Current management in child neurology. 3rd edn. Hamilton, Ontario, Canada: BC Decker, 2005: 93-8.

4. Ferrie CD. Daydreaming and childhood preoccupation. Journal of Paediatric Neurology 2010;8:57-8.

5. Mink JW, Neil JJ. Masturbation mimicking paroxysmal dystonia or dyskinesia in a young girl. Mov Disord 1995;10:518-20.

6. DiMario FJ. Movement disorders. In: DiMario FJ, ed. Non-epileptic childhood paroxysmal disorders. New York: Oxford University Press, 2009:217-93.

7. Evans RW, Rolak LA. The Alice in wonderland syndrome. Headache 2004;44:624-5.
8. Podoll K, Robinson D. Out-of-body experiences and related phenomenon in migraine art. Cephalgia 1999;19:886-96.

9. Sances G, Guaschino E, Perucca P, et al. Migralepsy: a call for a revision of the definition. Epilepsia 2009;50:2487-96.

10. Commission of Classification and Terminology of the international League Against Epilepsy. Proposal for revised clinical and electroencephalographic classification of epileptic seizures. Epilepsia 1981;22:489-501.

11. Panayiotopoulos CP. Idiopathic generalized epilepsies. In: Panayiotopoulos CP, ed. The epilepsies: seizures, syndromes and management. Oxford: Bladon Medical Publishing, 2005:271-348.

12. Dulac O. Atypical absences. http://www.ilae.org/ctf/ atypical_absence.html (accessed 10 Feb 2012).

13. Panayiotopoulos CP. Eyelid myoclonia with and without absences. http://www.ilae.org/visitors/centre/ctf/ eyelid_myoclonia_w_wo_abs.html (accessed 10 Feb 2012).

14. Jocic-Jakubi B, Jovanovic M, Jankovic DS, et al. Frontal-onset absences in children: associated with worse outcome? A replication study. Seizure 2009;18:275-8.

15. Commission on Classification and Terminology of the International League Against Epilepsy. Proposal for revised classification of epilepsies and epileptic syndromes. Epilepsia 1989;30:389-99.

16. Loiseau P, Panayiotopoulos CP. Childhood absence epilepsies. http://www.ilae.org/visitors/centre/ctf/childhood_absence.html (accessed 10 Feb 2012).

17. Panayiotopoulos CP. Typical absence seizures. http://www.ilae. org/ctf/typical_absence.html (accessed 10 Feb 2012).

18. Obeid T. Clinical and genetic aspects of Juvenile absence epilepsy. J Neurol 1994;241:487-91.

19. Oller L. Prospective study of the differences between the syndromes of infantile absence epilepsy and syndrome of juvenial absence epilepsy. Rev Neurol 1996;24:930-6.

20. Panayiotopoulos CP. Absence epilepsies. In: Engel JJ, Pedley TA. Epilepsy : a comprehensive textbook. Philadelphia: Lippincott-Raven Publishers, 1997:2327-46.

21. Rosenow F, Wyllie E, Kotagal P, et al. Staring spells in children: descriptive features distinguishing epileptic and nonepileptic events. J Pediatr 1998;133:660-3.

22. Foerster O. Hyperventilationsepilepsie. Dtsch Z Nervenheilkd 1924;83:347-56.

23. Wirrell E, Camfield P, Gordon K, et al. Will a critical level of hyperventilation-induced hypocapnia always induce an absence seizure? Epilepsia 1996;37:459-62.

24. Watemberg N, Tziperman B, Dabby R, et al. Adding video recording increases the diagnostic yield of routine electroencephalograms in children with frequent paroxysmal events. Epilepsia 2005;46:716-9.

25. National Institute for Clinical Excellence. The epilepsies: diagnosis and management of the epilepsies in children and young people in primary and secondary care. http://www.nice. org.uk/nicemedia/pdf/CG020childrenquickrefguide.pdf (accessed 10 Feb 2012).

26. Panayiotopoulos CP. Treatment of typical absence seizures and related epileptic syndromes. Paediatr Drugs 2001;3:379-403.

27. Glauser TA, Cnaan A, Shinnar S, et al. Ethosuximide, valproic acid and lamotrigine in childhood absence epilepsy. N Engl J Med 2010;362:790-9. 